\title{
Taking simvastatin in the morning compared with in the evening: randomised controlled trial
}

\author{
Alan Wallace, David Chinn, Greg Rubin
}

Grangewood

Surgery, Houghton

le Spring, Tyne an

Wear DH4 4RB

Alan Wallace

general practitioner

Centre for Primary and Community

Care, University of

Sunderland,

Benedict Building,

Sunderland, Tyne

and Wear SR2 7BW

David Chinn

senior research fellow

Greg Rubin

professor

Correspondence to:

A Wallace

Awallace40@

aol.com

BMJ 2003;327:788
Statins are widely prescribed for the primary and secondary prevention of coronary artery disease. They act by inhibiting the enzyme HMG CoA reductase, which controls synthesis of cholesterol in the liver. Most manufacturers of statins recommend that they are taken at night, on the basis of physiological studies which show that most cholesterol is synthesised when dietary intake is at its lowest. ${ }^{1}$ One small clinical trial found that taking smaller doses of simvastatin than are used in treatment, in the morning, was less efficient. ${ }^{2}$ However, a trial using atorvastatin found no significant difference in cholesterol concentrations between patients taking the statin in the morning and those taking it in the evening. ${ }^{3}$

Doubt has been cast on whether statins need to be taken at night, ${ }^{4}$ particularly as many patients also receive treatment with other cardioprotective drugs and compliance may be compromised by multiple dosing. ${ }^{5}$ We determined whether taking simvastatin in the morning had significantly different efficiency from taking it in the evening, by measuring cholesterol concentrations in fasting patients' serum.

\section{Participants, methods, and results}

We randomised adults stable on 10 or $20 \mathrm{mg}$ of simvastatin at night for primary or secondary prevention of coronary heart disease, stroke, or peripheral vascular disease, to dosings in the morning or evening for a period of eight weeks. We measured fasting blood lipid profiles at baseline and at eight weeks. We sampled blood between 830 and $930 \mathrm{am}$. Patients randomised to dosing in the morning were told not to omit it on the day of the follow up blood sample. The primary outcome measure was change in fasting total cholesterol concentration between baseline and follow up. We also measured the concentration of high density lipoprotein, alanine transaminase, low density lipoprotein, and triglyceride. We checked compliance by counting the number of tablets taken between the two visits.

We compared mean lipid concentrations between treatment groups in an intention to treat analysis using analysis of covariance with initial concentration as a covariate. We compared proportions with $\chi^{2}$ tests and means with unmatched $t$ tests. The $5 \%$ level indicated statistical significance, and we analysed data in SPSS.

Lipid concentrations at baseline (mean and standard deviation) with mean and $95 \%$ confidence interval of the difference in the change after eight weeks of morning versus evening dosing $(n=60)$

\begin{tabular}{lccc} 
& \multicolumn{2}{c}{ Mean concentration } & \\
\cline { 2 - 3 } Lipid & Baseline (SD) & Change $\mathbf{( 9 5 \% ~} \mathbf{~ I l})$ & P value $^{\text {* }}$ \\
\hline Total cholesterol (mmol/l) & $4.4(0.8)$ & $0.38(0.17$ to 0.59$)$ & 0.001 \\
\hline Low density lipoprotein $(\mathrm{mmol} / \mathrm{l})$ & $2.4(0.6)$ & $0.25(0.06$ to 0.44$)$ & 0.012 \\
\hline High density lipoprotein $(\mathrm{mmol} / \mathrm{l})$ & $1.3(0.3)$ & $0.02(-0.03$ to 0.08$)$ & 0.43 \\
\hline Triglyceride $(\mathrm{mmol} / \mathrm{l})$ & $1.6(0.8)$ & $0.09(-0.31$ to 0.48$)$ & 0.67 \\
\hline Alanine transaminase $(\mathrm{IU} / \mathrm{l})$ & $22.0(11.0)$ & $-1.32(-5.6$ to 3.0$)$ & 0.55 \\
\hline
\end{tabular}

${ }^{*}$ Analysis of covariance with adjustment for initial concentration.
To show a significant $4 \%$ change in mean cholesterol concentration with $80 \%$ power, assuming a mean coefficient of variation of $5.5 \%$ among patients, we needed 60 patients.

A computer search identified 96 patients who took simvastatin. We excluded 13 because they already took simvastin in the morning. Of the 83 eligible patients, $60(72 \%)$ accepted. We randomised the 27 men and 33 women of mean age 66 (range 44-82) to taking the drug in the mornings or in the evenings. Thirty six patients took $10 \mathrm{mg}$ a day and 24 took $20 \mathrm{mg}$ a day. Sex and daily dose did not differ between groups $(\mathrm{P}>0.6)$. A total of 57 patients completed the trial; one withdrew and two defaulted. In the intention to treat analysis, we assumed that the lipid profiles of these patients did not change between baseline and follow up. Change in blood lipids was normally distributed and switching taking simvastatin from in the evening to in the morning resulted in statistically significant increases in total and low density lipoprotein cholesterol (table). Compliance did not differ between the groups (mean number of tablets 1 , standard deviation 5 for both groups; $\mathrm{P}=0.9$ ).

\section{Comment}

Simvastatin is probably best taken at night because concentrations of total cholesterol and of low density lipoprotein are significantly greater when it is taken in the morning. Simvastatin is the most widely prescribed statin in the United Kingdom, and this finding has implications for compliance in preventing coronary heart disease. The absence of a similar effect with atorvastatin may be explained by its longer elimination half life

We thank Pauline Shaw and Glennis Lowdon for collecting data and blood samples.

Contributors: AW had the original idea, secured funding, and collected the data. DC and GR developed the methods, and DC analysed the data. All authors wrote the paper. AW is guarantor. Funding: Royal College of General Practitioners Scientific Foundation Board. AW was funded by a research training bursary from the Centre for Primary and Community Care, University of Sunderland.

Competing interests: None declared.

Ethical approval: Sunderland local research ethics committee. Miettinen TA. Diurnal variation of LDL and HDL cholesterol. Ann Clin
Res 1980;12:295-8.
Saito Y, Yoshida S, Nakaya N, Hata Y, Goto Y. Comparison between
morning and evening doses of simvastatin in hyperlipidemic subjects: a
double-blind comparative study. Arterioscler Thromb 1991;11:816-26.
Cilla DD, Gibson DM, Whitfield LR, Sedman AJ. Pharmacodynamic
effects of atorvastatin after administration to normocholesterolaemic
patients in the morning and evening.J Clin Pharmacol 1996;36:604-9.
4 Bandolier. Cholesterol fairy home to roost. Bandolier 2001;88:7.
www.jr2.ox.ac.uk/bandolier/band88/b88-7.html (accessed 15 Aug 2003). compliance with lipid-lowering drugs in hyperlipidemic patients. J Clin Pharm Ther 2000;25:445-51.

(Accepted 25 July 2003) 